\title{
An evidence-based, point-of-care tool to guide completion of asthma action plans in practice
}

\author{
Andrew Kouri ${ }^{1}$, Louis-Philippe Boulet ${ }^{2}$, Alan Kaplan ${ }^{3}$ and Samir Gupta ${ }^{1,4}$
}

Affiliations: ${ }^{1}$ Dept of Medicine, Division of Respirology, University of Toronto, Toronto, ON, Canada. ${ }^{2}$ Institut universitaire de cardiologie et de pneumologie de Québec, Université Laval, Québec, QC, Canada. ${ }^{3}$ Family Physician Airways Group of Canada, University of Toronto, Toronto, ON, Canada. ${ }^{4}$ The Keenan Research Centre in the Li Ka Shing Knowledge Institute of St Michael's Hospital, Toronto, ON, Canada.

Correspondence: Samir Gupta, St Michael's Hospital, Room 6044, Bond Wing, 30 Bond Street, Toronto, ON, M5B 1W8, Canada. E-mail: GuptaSAsmh.ca

\section{@ERSpublications}

New tool brings complex evidence to the point-of-care, guiding providers on how to create the best \#asthmaactionplan http://ow.ly/rmFu30a9neW

Cite this article as: Kouri A, Boulet L-P, Kaplan A, et al. An evidence-based, point-of-care tool to guide completion of asthma action plans in practice. Eur Respir J 2017; 49: 1602238 [https://doi.org/10.1183/ 13993003.02238-2016].

ABSTRACT Asthma action plans (AAPs) reduce healthcare utilisation, improve quality of life and are recommended across guidelines. However, fewer than $25 \%$ of patients receive an AAP, partly due to prescribers' inability to complete "yellow zone" instructions (how to intensify therapy for acute loss of control). We sought to review best evidence to develop a practical, evidence-based tool to facilitate yellow zone guidance in adults.

We reviewed recent asthma guidelines and adult studies addressing acute loss of asthma control (January 2010 to March 2016). We developed evidence-based rules for yellow zone therapy and operational guidelines to maximise adherence and minimise errors.

We reviewed three guidelines and 11 manuscripts (2486 abstracts screened). Recommendations were comparable but some areas lacked guidance. For 15/43 asthma regimens, the commonly recommended four- to five-fold yellow zone inhaled corticosteroid dose increase was problematic due to regulatory dose limits. We identified evidence-based alternatives for 8/15 regimens. Operational guidance included increasing to a maximum of four inhalations while maintaining baseline inhaler frequency and device in the yellow zone.

We developed a practical implementation tool to facilitate AAP delivery at the point of care, addressing existing gaps and uncertainties. Our tool should be implemented as part of a multifaceted approach to augment AAP usage.

This article has supplementary material available from erj.ersjournals.com

Earn CME accreditation by answering questions about this article. You will find these at erj.ersjournals.com/journal/cme Received: Nov 132016 | Accepted after revision: Feb 172017

The views expressed in this article are those of the authors and not an official position of the institution or funder.

Conflict of interest: None declared.

Copyright $\odot$ ERS 2017. This ERJ Open article is open access and distributed under the terms of the Creative Commons Attribution Non-Commercial Licence 4.0. 


\section{Introduction}

Asthma is one of the most common chronic diseases in Europe, is increasing in prevalence, and is projected to carry a healthcare expenditure of more than $€ 19$ billion [1]. Although effective therapies exist, up to $53 \%$ of patients remain poorly controlled $[2,3]$.

An asthma action plan (AAP) is an individualised written plan produced by a healthcare professional for a patient with asthma, providing education and guidelines for self-management of worsening symptoms in a "traffic light" configuration [4]. A "green zone" describes adequate control and corresponding baseline medications, a "yellow zone" describes loss of control and corresponding instructions for therapeutic intensification, and a "red zone" indicates severe symptoms that should prompt immediate medical assistance [5]. A Cochrane review of 36 randomised controlled trials (RCTs) demonstrated that use of a written AAP in conjunction with education and regular clinical review significantly reduces healthcare utilisation, absenteeism and asthma symptoms, and improves quality of life [4]. Furthermore, AAP use has been associated with a $70 \%$ reduction in mortality [6]. In accordance with this strong evidence, international asthma guidelines recommend that all patients receive a written AAP [7-9].

Despite these data, AAP provision and use remains poor. In a study examining patients in the European Community Respiratory Health Survey II, only 30\% of asthmatic patients who had used inhaled corticosteroid in the past year received a personalised AAP [10]. The principal barriers to AAP uptake are at the level of the provider and include lack of time, experience and confidence in creating appropriate AAP recommendations [11-13]. In one study, 30\% of motivated physicians attending an asthma skills workshop were unable to prepare an adequate AAP [13]. Although recent asthma guidelines attempt to address this knowledge gap by providing evidence-based recommendations for changes to therapy in the yellow zone of the AAP, practical implementation remains complex and no implementation tools are widely available $[7-9,14]$.

We sought to develop a practical, evidence-based point-of-care guide for populating AAP yellow zone instructions in adult patients (age $\geqslant 18$ years) with persistent asthma on regular maintenance therapy, with a goal of facilitating AAP delivery.

\section{Methods}

\section{Evidence review}

We used recommendations in international guidelines complemented by a literature search as the basis for our algorithm.

\section{Guideline review}

We searched major international asthma guidelines published in the last 5 years (prior to December 2016) for recommendations regarding AAP yellow zone formulation. We included guidelines produced by the Global Initiative for Asthma (GINA) [7], the British Thoracic Society/Scottish Intercollegiate Guideline Network (BTS/SIGN) [8] and the Canadian Thoracic Society (CTS) [9].

\section{Literature review}

The literature search in the oldest included guideline (CTS) was performed in October 2010. Accordingly, we searched for English-language studies involving human adult subjects, using asthma search terms as $\mathrm{MeSH}$ and keywords in Ovid MEDLINE, EMBASE and the Cochrane Central Registry of Controlled Trials from January 2010 to March 2016 (a complete list of search terms can be found in appendix 3). We included all RCTs, reviews and systematic reviews that described strategies for adjusting controller therapy for acute loss of asthma control in adults with persistent asthma on regular maintenance therapy. A. Kouri and S. Gupta reviewed all abstracts and categorised them as definitely, possibly or definitely not meeting inclusion criteria, and then reviewed full manuscripts for all abstracts definitely or possibly meeting inclusion criteria to determine whether they should be included. Any differences were settled by discussion, leading to consensus.

We examined all citations of interest from reference lists of retrieved articles and used a reference harvesting approach on all included papers, using Web of Science and Google Scholar, to identify any additional relevant publications.

We searched all included publications for guidance in areas where guideline recommendations were lacking or divergent and for new evidence.

\section{Algorithm development}

Based on this review, we established evidence-based rules for therapeutic intensification in the yellow zone. In cases where guideline recommendations and/or literature search findings diverged, we reviewed original 
studies cited by guideline writers to determine which approach was best supported. Next, we attempted to apply these rules to a catalogue of common controller medication dose and frequency regimens (for each commercially available medication). We documented regulatory limits on total daily doses for each medication according to international drug licensing agencies (appendix 2, table S1). In cases where yellow zone doses exceeded recommended daily limits across agencies, we searched guideline references and our search results and developed evidence-based alternative approaches where possible. Given that more than one medication type, dose and/or frequency could be used to operationalise yellow zone instructions, we developed practical rules to simplify and standardise prescriptions while maximising patient adherence and minimising patient errors. These rules were based on best evidence where available and on expert opinion where no evidence was available. Relevant expertise included asthma guideline development (A. Kaplan, L-P. Boulet), primary care practice (A. Kaplan) and guideline implementation (L-P. Boulet, S. Gupta).

\section{Results}

Evidence review

\section{Guideline review}

Included guidelines provided mostly comparable recommendations for self-management of acute loss of asthma control using an AAP (table 1).

\section{Literature review}

We identified 2486 abstracts between January 2010 and March 2016. Of these, 51 (2\%) possibly met and 2435 (98\%) did not meet inclusion criteria. Upon full manuscript review, 11 (22\%) of 51 citations "possibly meeting inclusion criteria" met inclusion criteria. We did not identify any additional citations of interest from the manual search or from reference harvesting. The 11 included reports consisted of 1 RCT, 1 practice guideline, 3 narrative reviews and 6 systematic reviews. Upon detailed analysis, the RCT was not applicable to an outpatient AAP, as it tested the effects of a nebulised inhaled corticosteroid against systemic corticosteroids [15]. The practice guideline and 8 of the 9 reviews had either been directly cited in one or more of the guidelines we reviewed, or all individual studies included in the publication had been cited in one or more guidelines. Accordingly, where relevant, data from these studies had already been accounted for in our algorithm. However, the remaining review cited a relevant trial that had not been referenced in the included guidelines, comparing high-dose ciclesonide to prednisone for acute loss of asthma control. Although the dose used exceeded regulatory limits for total daily dosing, the study demonstrated both equivalence and safety and its findings were therefore incorporated into our algorithm [16]. See appendix 2 table S2, for full discussion of reviewed studies.

\section{Algorithm development}

For inhaled corticosteroid (ICS) monotherapy, the CTS and BTS/SIGN guidelines were consistent, recommending increasing the ICS dose by a factor of four to five (with strong levels of evidence; table 1) $[8,9]$. The BTS/SIGN guideline limited its recommendation to patients on a low baseline ICS dose $(200 \mu \mathrm{g}$ beclomethasone diproprionate (BDP) equivalent). However, referenced studies included patients on moderate and high baseline ICS doses, suggesting that this practice should apply to various dose levels. We do note that a single trial demonstrated that immediate doubling of ICS dose in response to a drop in peak expiratory flow (PEF) can reduce asthma exacerbations [17]. However, this finding has not been replicated and most patients do not routinely monitor PEF. Although more evidence is required in this area, the balance of existing evidence favours increasing the ICS dose by four- to five-fold.

For non-ICS/formoterol combination therapy, CTS guidelines recommended taking a similar approach to that in patients on ICS alone (quadrupling the ICS component), whereas GINA guidelines recommended increasing the ICS component without specific dose guidance (except a maximum ICS dose of $2000 \mu \mathrm{g}$ BDP equivalent). Both groups acknowledged a dearth of evidence in this area and made these recommendations based on consensus $[7,9]$. We adopted the CTS approach, which provides a pragmatic rule for yellow zone dose intensification. In one exceptional circumstance, we recommended simply quadrupling the dose of a fluticasone furoate/vilanterol combination inhaler, as vilanterol doses of up to $100 \mu \mathrm{g}$ have been shown to be safe for short courses of therapy [18, 19].

For ICS/formoterol therapy using the adjustable maintenance dosing (AMD) approach, GINA guidelines recommended quadrupling the maintenance ICS/formoterol dose (to a maximum of $72 \mu \mathrm{g} \cdot \mathrm{day}^{-1}$ formoterol), whereas CTS guidelines recommended increasing the number of puffs to a maximum of 4 puffs twice daily $\left(48 \mu \mathrm{g} \cdot \mathrm{day}^{-1}\right.$ formoterol) $[7,9]$. These slightly divergent recommendations were based on a nearly identical set of studies (GINA guidelines cited a 2006 review by REDDEL et al. [20], which included six trials, whereas CTS guidelines cited a 2007 review by EDwARDS et al. [21] which included the same six trials and an additional five trials) and assigned similarly high levels of evidence (table 1). However, all cited studies compared yellow zone dose intensification in patients on budesonide/formoterol-AMD to a 
TABLE 1 Guideline recommendations for managing acute loss of asthma control in adults using an asthma action plan (AAP)

\begin{tabular}{cc} 
& BTS/SIGN 2016 [8] \\
\hline $\begin{array}{c}\text { Criteria for initiating increase in } \\
\text { controller medication }\end{array}$ & $\begin{array}{c}\text { Personalised action plans should be } \\
\text { triggered by symptoms and/or peak }\end{array}$ \\
("activating the yellow zone") & flows (PEF between 60 and $80 \%$ best \\
and duration of change & suggested for increase in inhaled \\
& corticosteroid). Timing and duration \\
of change not specified
\end{tabular}

How to adjust ICS monotherapy during acute loss of asthma control

In adult patients on a very low dose of ICS, a five-fold increase in dose at the time of an asthma attack leads to a decrease in the severity of asthma attacks. Duration of change not specified.

Doubling the dose of ICS at the time of an exacerbation is of unproven value.

Level of evidence: Level 1+ ${ }^{\#}$

No specific recommendations. combination medications during acute loss of asthma control lother than ICS/ formoterol combination medications)

How to adjust ICS/formoterol combination medications (used as AMD or MART) during acute loss of asthma control

\section{Level of evidence: N/A}

AMD: No specific recommendations pertaining to AAPs.

MART: No specific recommendations pertaining to AAPs.

\section{GINA 2016 [7]}

When there is a clinically importan change from the patient's usual level of asthma control, for example, if asthma symptoms are interfering with normal activities, or PEF has fallen by $>20 \%$ for more than 2 days. Duration of change $1-$ 2 weeks

At least double ICS; consider increasing ICS to high dose (maximum $2000 \mu \mathrm{g} \cdot$ day $^{-1}$ BDP equivalent].

Level of evidence: Evidence $B^{\text {वा }}$

Step up to higher dose formulation of ICS/LABA, or consider adding a separate ICS inhaler (to maximum total $2000 \mu \mathrm{g} \cdot$ day $^{-1}$ BDP equivalent).

Level of evidence: N/A

Instructions for patients who do not respond to first-line therapy lincreases in their controller therapy) during acute loss of asthma control

\section{Alternative action plan instructions for specific patient populations unlikely to benefit from increases in inhaled controller medications alone during acute loss of asthma control}

Patients may safely hold an emergency supply of prednisolone tablets for use if their symptoms continue to deteriorate and/or if their peak flow falls to $60 \%$ of their best. Recommend prednisolone 40-50 mg daily for at least 5 days or until recovery.

Level of evidence: Level 1++

In patients already taking moderate or high doses $1 \geqslant 400 \mu \mathrm{g} \cdot$ day $^{-1}$ BDP equivalent dailyl of inhaled corticosteroids, begin oral steroids (prednisolone $40-50 \mathrm{mg}$ daily for at least 5 days or until recoveryl.

Level of evidence: N/A
Level of evidence: Level $D^{\text {f }}$

AMD: quadruple maintenance dose (maximum formoterol $72 \mu \mathrm{g} \cdot$ day $^{-1}$ ).

MART: continue maintenance dose and increase reliever use as needed (maximum formoterol total $72 \mu \mathrm{g} \cdot$ day $^{-1}$ ).

Level of evidence: AMD: Level $B^{\text {व }}$ MART: Level $A^{\text {q }}$ increase in reliever and controller medication for 2-3 days or deteriorate rapidly should take prednisone $1 \mathrm{mg} \cdot \mathrm{kg}^{-1} \cdot \mathrm{day}^{-1}$ (maximum $50 \mathrm{mg}$ ) for 5-7 days.

Level of evidence: Level $A^{\text {I }}$

In patients who present with PEF or $\mathrm{FEV}_{1}<60 \%$ of their personal best or predicted value, or have a history of sudden severe exacerbations, start prednisone $1 \mathrm{mg} \cdot \mathrm{kg}^{-1} \cdot \mathrm{day}^{-1}$ (maximum $50 \mathrm{mg}$ ) for 5-7 days.

Level of evidence: Level $A^{\text {n }}$
Patients who fail to respond to an

\section{CTS 2012 [9]}

Action plans should outline when and how to adjust reliever and controller therapy for loss of control. Specific symptoms, or PEF thresholds and timing for increasing controller medication not provided. Duration of change 1-2 weeks

Increase the ICS dose by four- or five-fold for 7-14 days in adults with a history of severe exacerbations in the past year requiring systemic steroids.

Level of evidence: Grade $2 \mathrm{C}^{+}$

Fourfold or greater increase in ICS dose for 7-14 days in individuals who are exacerbation-prone.

Level of evidence: Consensus ${ }^{+}$

AMD: increase to a maximum of four inhalations twice daily for 7-14 days. MART: continue use in lieu of increasing the ICS dose of fixed-dose ICS/LABA combination therapy.

Level of evidence: AMD: Grade 2B ${ }^{+}$ MART: Grade 2B ${ }^{+}$

No specific recommendations.

Level of evidence: N/A

In individuals over the age of 15 years with a history of severe acute loss of asthma control in the preceding year, start prednisone $30-50 \mathrm{mg}$ daily for at least 5 days.

Level of evidence: N/A

\#: 1++ indicates high quality meta-analyses, systematic reviews or randomised controlled trials (RCTs), or RCTs with a very low risk of bias; $1+$ indicates well-conducted meta-analyses, systematic reviews, or RCTs with a low risk of bias (British Thoracic Society/Scottish Intercollegiate Guideline Network (BTS/SIGN) recommendation ranking system). ": Level A: recommendation from RCTs, from a rich body of data, from well-designed studies with a consistent pattern of findings, from a large number of studies with a substantial number of patients. Level B: recommendation from RCTs, with limited body of data, from studies with limited number of patients, from subgroup or post-hoc analyses, or meta-analyses of RCTs or studies with inconsistent results. Level D recommendation from expert opinion, based on clinical experience or knowledge that cannot be included in category C. (Global Initiative for Asthma (GINA) evidence levels.) ${ }^{+}: 2 B$ is a weak recommendation with moderate quality of evidence. $2 \mathrm{C}$ is a weak recommendation with low quality of evidence. Consensus recommendation implies lack of evidence, where a recommendation is made based on expert opinion and practice experience. (GRADE system). CTS: Canadian Thoracic Society; ICS: inhaled corticosteroid; LABA: long-acting beta-agonist; AMD: adjustable maintenance dosing with ICS/formoterol combination inhalers; MART: maintenance and reliever therapy using an ICS/formoterol combination inhaler; PEF: peak expiratory flow; BDP: beclomethasone diproprionate; FEV1: forced expiratory volume in $1 \mathrm{~s} ; \mathrm{N} / \mathrm{A}$ : not available. 
higher baseline fixed dose of budesonide/formoterol. Accordingly, they concluded that yellow zone dose intensification is preferred to a higher baseline dose (with no change in the yellow zone), but did not answer the question as to which approach would be preferred in the yellow zone. A lack of available data to answer this specific question was noted by CTS guideline writers [9]. Practically, for typically recommended starting dose levels (budesonide/formoterol 100/6 $\mu \mathrm{g}$ or 200/6 $\mu \mathrm{g} 1$ puff twice daily), GINA and CTS recommendations amounted to the same yellow zone change. Their recommendations diverge in patients on unconventional dose regimens (e.g. 100/6 $\mu \mathrm{g} 3$ puffs twice daily or 200/6 $\mu \mathrm{g} 2$ puffs twice daily). In these cases, following the CTS recommendation to increase to 4 puffs twice daily would equate to doubling (or less) the total ICS dose, which guidelines universally discourage. We therefore adopted the GINA guideline recommendation to quadruple the maintenance ICS/formoterol dose (table 2).

For ICS/formoterol therapy using the MART (maintenance and reliever therapy) approach, GINA and CTS guidelines recommended continuing this approach in the yellow zone, with a consistently high level of evidence $[7,9]$.

Guidelines were consistent in their recommendation that a short course of oral corticosteroids (OCS) should be considered as second-line therapy in the yellow zone when initial increases in inhaled therapy are not effective and/or if a clinical deterioration occurs (table 1) [7, 8]. We adopted specific criteria provided by all guidelines for such use of OCS (table 2). GINA and CTS guidelines also recommended considering OCS as first-line therapy in patients with certain high-risk features (table 1). Accordingly, we added these caveats to our yellow zone recommendation table (table 2). It should be noted that an AAP that includes conditional recommendations for ICS dose increases followed by addition of an OCS can be confusing to some patients. To address this, OCS recommendations could be operationalised by providing criteria for patients to contact their provider for an OCS prescription, while reserving a standing prescription with a conditional self-management plan only for patients with excellent self-management skills (table 2, footnotes). Also, although we have included the option to add OCS after failed initial ICS intensification as part of the yellow zone, some AAPs have operationalised this through an extra "orange zone" and others by including OCS in the "red zone" [22, 23]. This recommendation should thus be operationalised in accordance with the particular AAP format that the clinician is using.

Finally, certain patients were on baseline ICS doses that did not permit a four- to five-fold dose increase without exceeding regulatory limits for the total daily dose. In such patients on fluticasone, budesonide or ciclesonide, we were able to make evidence-based dose increase recommendations guided by specific studies demonstrating the safety and efficacy of dose increases (see section "Additional rules"). However, for other medications where such data are lacking, we offered two options: either temporarily exceeding regulatory limits by increasing the ICS dose four-fold, or going straight to a short course of OCS. Although we concede that having two options adds complexity, there are important considerations that lend support to each approach, leading to a conclusion that the therapeutic decision in this situation requires individualisation. Considerations favouring exceeding regulatory dose limits with ICS include that 1) regulatory limits on total daily ICS doses are intended for chronic use and not short-term use as part of step-up therapy in an AAP; 2) short-term use of doses exceeding regulatory limits has been shown to be safe in other medication preparations (ciclesonide in particular) [16]; and 3) one of the main goals of AAPs is to avoid a requirement for systemic steroids, which have several documented risks and side-effects. Considerations favouring going straight to a short course of OCS include that 1) pharmacists and patients often object to dispensing and consuming medications at doses that exceed product monograph specifications; 2) safety data for short-term higher dose usage are not available for these medications; and 3) many primary-care clinicians may not feel comfortable writing prescriptions that exceed regulatory dose limits, thus limiting the practical usability of such a recommendation in real-world care.

\section{Additional rules}

In cases where above rules could not be implemented due to maximum daily ICS dose limits (appendix 2, table S1), we developed additional evidence-based rules where applicable (table 2).

\section{High-dose fluticasone}

For patients on a baseline daily fluticasone dose greater than $500 \mu \mathrm{g}$ (ICS alone or in a combination inhaler), a four- or five-fold dose increase would exceed the regulatory limit of a total daily dose of $2000 \mu \mathrm{g}$. However, two studies suggest that a four- to five-fold intensification might not be required in these patients. LeVy et al. [24] and Di FRANCO et al. [25] compared fluticasone $2000 \mu \mathrm{g} \cdot$ day $^{-1}$ (with or without a long-acting beta-agonist (LABA)) to a course of OCS in patients presenting to the emergency department with asthma exacerbation severe enough to warrant systemic steroids. Given a mean baseline dose of approximately $800 \mu \mathrm{g}$ BDP-equivalent (equivalent to $800 \mu \mathrm{g}$ fluticasone [9]), this represented an approximately 2.5 -fold increase. Both trials found high-dose inhaled fluticasone to be non-inferior to OCS [24, 25]. Baseline doses were 
TABLE 2 Proposed evidence-based instructions for managing acute loss of asthma control in adults using an asthma action plan

\section{Baseline controller medication (green zone)}

\section{Recommended changes during acute loss of asthma} control (yellow zone)

\section{ICS monotherapy}

What to do if a four- to five-fold increase exceeds the daily dose limit

Fluticasone monotherapy at $>500 \mu \mathrm{g}$ total daily dose

Budesonide monotherapy at $>600 \mu \mathrm{g}$ total daily dose

Ciclesonide monotherapy at $>200 \mu$ total daily dose

Other medications

\section{Fixed dose ICS/LABA combination therapy}

What to do if a four- to five-fold increase exceeds the daily dose limit

Fluticasone/salmeterol at $>500 \mu \mathrm{g}$ fluticasone total daily dose Budesonide/formoterol at $>600 \mu \mathrm{g}$ budesonide total daily dose

Other medications

\section{ICS/formoterol combination therapy using MART approach}

Special clinical scenarios

Patients with a history of sudden and severe exacerbations and/or presenting with PEF or FEV $1 \leqslant 60 \%$ of personal best/predicted (severe exacerbation)

Patients who fail to improve clinically within 2-3 days of increase in controller medication, and/or have a rapid clinical deterioration, and/or have a PEF or FEV1 that falls to $\leqslant 60 \%$ of their personal best value
Increase the daily ICS dose by four- to five-fold for 7-14 days, provided the dose does not exceed the regulatory limit on total daily dose

Increase the total daily fluticasone dose to $2000 \mu \mathrm{g} \cdot$ day $^{-1}$ for $7-14$ days

Increase the total daily budesonide dose to $2400 \mu \mathrm{g} \cdot$ day $^{-1}$ for 7-14 days

Increase total daily ciclesonide dose to $1600 \mu \mathrm{g} \cdot$ day $^{-1}$ for $7-14$ days ${ }^{\#}$

Option 1: increase the daily ICS dose by four- to five-fold for 7-14 days, temporarily exceeding the regulatory limit on total daily dosen

Option 2: prednisone $30-50 \mathrm{mg}$ daily (or equivalent OCS regimen) for $5-7$ days $^{+}$

Increase the daily ICS dose by four- to five-fold for 7-14 days, provided the dose does not exceed the regulatory limit on total daily dose of ICS ladd ICS alone unless increase of ICS/LABA possible without surpassing regulatory limit on total daily dose of LABAl

Increase the total daily fluticasone dose to $2000 \mu \mathrm{g} \cdot$ day $^{-1}$ for 7-14 days (by adding fluticasone)

Increase the total daily budesonide dose to $2400 \mu \mathrm{g} \cdot$ day $^{-1}$ for 7-14 days (by adding budesonide)

Option 1: increase the daily ICS dose by four- to five-fold for 7-14 days, temporarily exceeding the regulatory limit on total daily dose (by adding an ICS to the ICS/LABA therapy)

Option 2: prednisone $30-50 \mathrm{mg}$ daily (or equivalent OCS regimen) for $5-7$ days $^{+}$

Continue MART therapy as prescribed

Prednisone $30-50 \mathrm{mg}$ daily (or equivalent OCS regimen) for $5-7$ days ${ }^{+}$

Prednisone $30-50 \mathrm{mg}$ daily (or equivalent OCS regimen) for $5-7$ days $^{+}$

\footnotetext{
\#: this dose exceeds regulatory limits (across jurisdictions) for routine daily use but has been shown to be safe and effective for treatment of acute loss of asthma control, in a clinical trial. ": total daily dose limits are intended for chronic daily use and a short-term dose increase beyond these limits is unlikely to carry any significant safety risks. However, formal safety testing data are not available and the decision to pursue this approach should be based on patient and clinician comfort. ${ }^{+}$: in patients with adequate experience self-managing their asthma, consider providing a standing prescription for oral corticosteroid (OCS) for these situations, with instructions to contact the primary healthcare provider after initiating OCS. For others, advise contacting their physicians to obtain an OCS prescription if they meet the criteria outlined in the asthma action plan. Ensure that all patients are appropriately counselled about the risks of short-term OCS use. ${ }^{\S}$ : fluticasone furoate/vilanterol inhaled corticosteroid (ICS)/long-acting beta-agonist (LABA) therapy can simply be quadrupled. Although the resulting vilanterol dose (100 $\mu \mathrm{g}$ ) daily would exceed regulatory limits, this has been shown to be safe for short courses of therapy (see text for references). MART: ICS/formoterol as maintenance and reliever therapy; PEF: peak expiratory flow; FEV1: forced expiratory volume in $1 \mathrm{~s}$.
}

highly variable and did not predict treatment failure [24]. These data suggest that increasing the total daily fluticasone dose to $2000 \mu \mathrm{g} \cdot \mathrm{day}^{-1}$ has a similar efficacy to a course of OCS, regardless of baseline ICS dose (see appendix 4 for further trial details). Given that patients who are self-managing through the yellow zone of an AAP would be expected to have less severe symptoms than patients seen in the emergency room, an increase to $2000 \mu \mathrm{g} \cdot \mathrm{day}^{-1}$ of fluticasone could be a reasonable alternative to OCS in patients whose baseline 
fluticasone dose is greater than $500 \mu \mathrm{g} \cdot \mathrm{day}^{-1}$. GINA guidelines also indicated that high-dose ICS has an equivalent effect to a short course of OCS (table 1).

Moderate- and high-dose budesonide

For patients on a baseline daily budesonide dose greater than $600 \mu \mathrm{g}$ (ICS alone or in a combination inhaler), a four- or five-fold dose increase would exceed the regulatory limit on total daily dose of $2400 \mu \mathrm{g}$. However, trials by FitzGerald et al. [26] and NANA et al. [27] demonstrated non-inferiority between high-dose budesonide and OCS in patients discharged from the emergency room after an asthma exacerbation (see appendix 4 for further trial details). As above, these data suggest that increasing the total daily budesonide dose to $2400 \mu \mathrm{g} \cdot \mathrm{day}^{-1}$ may have a similar efficacy to a course of OCS and presents a reasonable yellow zone option in patients with a baseline budesonide dose exceeding $600 \mu \mathrm{g} \cdot \mathrm{day}^{-1}$.

\section{High-dose ciclesonide}

For patients on a baseline daily ciclesonide dose of greater than $200 \mu \mathrm{g}$, a four- or five-fold increase would exceed the regulatory total daily dose limit of $800 \mu \mathrm{g}$. However, a trial by VAN DEN BERGE et al. [16] found that in 130 patients with worsening asthma, a 2 week course of ciclesonide $800 \mu \mathrm{g}$ twice daily was similar in efficacy to prednisolone $40 \mathrm{mg}$ daily for improvement in FEV1, morning PEF and symptoms. Importantly, no serious adverse events occurred with this high dose of ciclesonide and there were fewer overall adverse events than with prednisolone. These data suggest that ciclesonide can be safely and effectively increased past its regulatory total daily dose limit, allowing for quadrupling in patients on high baseline doses.

It should also be noted that exact symptom- and peak expiratory flow-based criteria, as well as the timing for initiating an increase in controller medications ("activating the yellow zone") during acute loss of asthma control were not specifically detailed in each guideline (table 1) and have been shown to be somewhat variable across existing AAPs $[23,28]$. However, based on RCTs demonstrating the efficacy of a four- to five-fold increase in ICS dose $[29,30]$, we recommend that patients initiate yellow-zone therapy within $24-48 \mathrm{~h}$ of loss of control.

\section{Practical implementation}

We established basic principles for formulating yellow zone prescriptions. These principles aim to establish a consistent approach while maximising patient adherence and minimising patient errors, applying best evidence where available and expert opinion where evidence is lacking (table 3).

Finally, we applied our algorithm and practical guidelines to a collection of common controller medication types and regimens, creating a yellow zone dose escalation chart that can be printed and posted in the clinical environment for practical use (printable charts, by region, in appendix 1).

\section{Discussion}

We have used best evidence from the latest international asthma guidelines and an updated literature review to create a practical algorithm for populating asthma action plans. Recommendations for intensifying controller therapy during acute loss of asthma control were broadly similar across major international guidelines, with more variance in areas lacking evidence. Of interest, our guideline review revealed that different international groups ascribed noticeably different levels of evidence to similar recommendations (table 1). For example, for ICS monotherapy, BTS/SIGN guidelines recommended a five-fold dose increase as a level $1+$ recommendation (corresponding to well-conducted meta-analyses, systematic reviews or RCTs with a low risk of bias), whereas CTS guidelines recommended a four- to five-fold increase as a level $2 \mathrm{C}$ recommendation (a weak recommendation with low-quality evidence). Our literature review did not find any significant new contributory studies published between the dates of the CTS and BTS guideline literature searches (appendix 2, table S2). Accordingly, variation probably resulted from differences in methodology, including evidence selection, synthesis, appraisal and recommendation formulation [37].

In attempting to apply guideline recommendations to develop a practical algorithm for use in a real-world clinical environment, we found several areas where recommendations were lacking or offered guidance that was difficult to operationalise. For example, for baseline regimens where intensification approaches were not supported by strong evidence (e.g. non-ICS/formoterol ICS/LABA medications), BTS/SIGN guidelines did not offer guidance (table 1). Additionally, even unanimously agreed-upon recommendations, such as increasing ICS doses by four- to five-fold, could not be universally applied due to regulatory limits on total daily doses. It would be infeasible to apply this recommendation in 15 of the 43 common dose regimens included in our practical chart (Appendix 1, Europe; our literature search identified alternative approaches for eight of these 15 cases). These factors support the commonly reported primary care perception that expert guidelines are too ambiguous [38] and rigid for application in individual patients [39, 40]. This 
TABLE 3 Proposed practical guidelines for adjusting maintenance medications during acute loss of asthma control

Recommendation

Maintain the controller medication as part of the "yellow zone" dose, where possible

Add additional inhalations while maintaining the baseline controller medication frequency, where possible

Increase the number of inhalations of the baseline controller medication to attain the target dose rather than prescribing an additional inhaler, where possible

When it is necessary to add a new inhaler in the "yellow zone," use the same device type as the baseline controller medication

Do not exceed 4 inhalations per use, and in cases where it is necessary to add a new puffer in the yellow zone, use a dose which favours the least number of inhalations per use

Consider patient-relevant issues such as medication cost and product shelf-life, particularly when proposing a requirement for new prescriptions in the "yellow zone"
Rationale

Reinforces that "green zone" controller medications are never to be stopped and minimises chances of the patient continuing with the incorrect puffer after yellow zone treatment is complete

Minimises dosing frequency, maintains consistency with the controller regimen, thereby minimising medication errors and maximising adherence

Minimises cost and complexity of filling a new inhaler prescription and maximises adherence

Maintains consistency with the baseline controller medication, thereby minimising medication administration errors and maximises adherence

Ensures practical applicability of yellow zone regimen, thereby maximising adherence

Excessive cost of treatment and the possibility of medication waste may negatively affect adherence

\section{Supporting evidence}

Adherence shown to decrease when maintenance medications are changed [31, 32]

Adherence shown to be inversely correlated with dosing frequency $[33,34]$

Adherence shown to decrease when new inhalers are added or more than one inhaler is recommended $[35,36]$

Adherence and asthma control shown to worsen when device type is changed [31]

MDI and DPI inhalers require up to $1 \mathrm{~min}$ per puff [36] More than 4 min per use is not practical and may decrease adherence lexpert opinion)

Adherence has been shown to be inversely correlated with socioeconomic status and income in some studies [36]

MDI: metered dose inhaler; DPI: dry powder inhaler.

poor "usability" is a consistently identified barrier to recommendation implementation [41, 42] and may be a factor in the observed low AAP usage rates in primary care [43].

Another important practical consideration is that recommended yellow zone doses could be achieved in various ways, including changes in the number and/or frequency of inhalations and/or through addition of a new inhaler or temporary replacement with a new inhaler. Despite this, guidelines did not specify how dose intensifications should be operationalised. A lack of any clear published guidance in this area probably also explains the large variability in yellow zone instructions across existing AAPs [23]. Given that both knowledge of available medication doses and formulations and time are major barriers to AAP usage in busy primary care environments, this uncertainty may also contribute to poor uptake [12, 13]. We attempted to address this by developing a series of practical implementation guidelines based on evidence and expert opinion. Our approach sought to minimise patient confusion and dosing errors while maximising patient satisfaction and adherence - notoriously difficult factors to elicit and to prioritise in guideline production [44]. This approach seeks not only to improve adherence to AAPs among patients, but also to address clinicians' concerns that patients may not effectively follow their AAPs, which in itself is a barrier to AAP delivery [45].

Our work has several limitations. As noted, our principal recommendations were based on major international guidelines, but our exception rules were based on small numbers of individual studies. It should be noted, however, that the evidence for AAP yellow zone formulation remains limited, and most guideline recommendations were also based on a small number of studies or expert consensus. Some of our practical rules for dose intensification regimens also relied on expert opinion and common sense, when evidence was lacking. Although we believe that this was necessary in order to produce a usable implementation tool, we recognise that more research is required to support our approaches in some of these areas. Our rules also do not provide guidance in patients with mild asthma who are not on regular controller therapy. Unfortunately, a literature review has found insufficient evidence to provide a recommendation for 
such patients [9]. We also appreciate that ensuring adherence to ICS is often challenging due to ICS aversion among patients [46] and that recommendations to further increase doses by four- to five-fold as part of an AAP will require patient education at the time of AAP delivery. Finally, we did not directly involve patients in developing these rules, which could have provided an important perspective.

We provide easy-to-follow, printable, paper-based algorithms that can be posted in a clinical setting, to inform completion of the AAP yellow zone (one each for Europe, Canada and the USA, in appendix 1). However, given that time is another major barrier to AAP delivery [3, 12, 47], integration of this algorithm into an electronic clinical decision support system would enable automated AAP generation, addressing both knowledge and time barriers to AAP delivery at the point of care. Accordingly, our team developed the Electronic Asthma Management System (eAMS), which features a patient questionnaire completed through a smartphone, tablet or PC interface in advance of the clinical visit, followed by real-time EMR (electronic medical record)-integrated advice for clinicians regarding asthma control status and corresponding medication adjustments, along with a pre-populated, personalised AAP (all content and recommendations can be altered by clinicians if required). A clinical trial of this system is currently under way.

In summary, we have developed a practical implementation tool to facilitate AAP delivery at the point of care, addressing the gaps and uncertainties in existing international guidelines. This tool addresses physician-level knowledge barriers as well as patient-level barriers surrounding adherence. However, other important barriers, including physician time and access to AAPs and patient aversion to steroid medications remain important determinants of AAP adherence. Our tool could be used as a paper reference guide or integrated into an electronic medical record system at the point of care. A future study should measure its impact on the frequency of AAP delivery, the quality of AAPs, patient uptake and patient-level outcomes. Future interventions should use our tool as part of a multifaceted approach to address the multiple known barriers to AAP usage.

\section{Acknowledgements}

We thank Len Fromer (Dept of Family Medicine, University of California at Los Angeles) and Kevin Gruffydd-Jones (University of Aberdeen) for support in developing the region-specific practical implementation tables. We also thank Suzanne Levitz, Robert Hauptman, David Cheah and Renu Bala Gupta for help piloting our tables for primary care physicians.

\section{References}

1 Accordini S, Corsico AG, Braggion $\mathrm{M}$, et al. The cost of persistent asthma in Europe: an international population-based study in adults. Int Arch Allergy Immunol 2013; 160: 93-101.

2 Chapman KR, Boulet LP, Rea RM, et al. Suboptimal asthma control: prevalence, detection and consequences in general practice. Eur Respir J 2008; 31: 320-325.

3 FitzGerald JM, Boulet L-P, McIvor RA, et al. Asthma control in Canada remains suboptimal: The Reality of Asthma Control (TRAC) study. Can Respir J 2006; 13: 253-259.

4 Gibson PG, Powell H, Wilson AJ, et al. Self-management education and regular practitioner review for adults with asthma. Cochrane Database Syst Rev 2003; CD001117.

5 Gupta S, Wan FT, Hall SE, et al. An asthma action plan created by physician, educator and patient online collaboration with usability and visual design optimization. Respiration 2012; 84: 406-415.

6 Abramson MJ, Bailey MJ, Couper FJ, et al. Are asthma medications and management related to deaths from asthma? Am J Respir Crit Care Med 2001; 163: 12-18.

7 GINA Executive Committee. Global Strategy for Asthma Management and Prevention, Global Initiative for Asthma (GINA) 2016. www.ginasthma.org Date last accessed: December 20, 2016.

8 British Thoracic Society/Scottish Intercollegiate Guidelines Network. British Guideline on the Management of Asthma: A National Clinial Guideline 2016. www.brit-thoracic.org.uk Date last accessed: December 20, 2016.

9 Lougheed MD, Lemiere C, Ducharme FM, et al. Canadian Thoracic Society 2012 guideline update: diagnosis and management of asthma in preschoolers, children and adults. Can Respir J 2012; 19: 127-164.

10 Cazzoletti L, Marcon A, Janson C, et al. Asthma control in Europe: a real-world evaluation based on an international population-based study. J Allergy Clin Immunol 2007; 120: 1360-1367.

11 Boulet L-P, Becker A, Bowie D, et al. Implementing practice guidelines: a workshop on guidelines dissemination and implementation with a focus on asthma and COPD. Can Respir J 2006; 13: Suppl. A, 5-47.

12 To T, McLimont S, Wang C, et al. How much do health care providers value a community-based asthma care program? A survey to collect their opinions on the utilities of and barriers to its uptake. BMC Health Serv Res 2009; 9: 77.

13 Lougheed MD, Moosa D, Finlayson S, et al. Impacts of a provincial asthma guidelines continuing medical education project: The Ontario Asthma Plan of Action's Provider Education in Asthma Care Project. Can Respir J 2007; 14: 111-117.

14 Pinnock H, Thomas M. Does self-management prevent severe exacerbations? Curr Opin Pulm Med 2015; 21: 95-102.

15 Go J, Ong E, Koh A. Comparison of intravenous hydrocortisone versus inhaled flulticasone in adult acute asthma: a randomized controlled trial. Respirology 2010; 15: 62.

16 Van den Berge M, Arshad SH, Ind PW, et al. Similar efficacy of ciclesonide versus prednisolone to treat asthma worsening after steroid tapering. Respir Med 2009; 103: 1216-1223.

17 Lahdensuo A, Haahtela T, Herrala J, et al. Randomised comparison of guided self management and traditional treatment of asthma over one year. BMJ 1996; 312: 748-752.

18 Lotvall J, Bateman ED, Bleecker ER, et al. 24-h duration of the novel LABA vilanterol trifenatate in asthma patients treated with inhaled corticosteroids. Eur Respir J 2012; 40: 570-579. 
19 Kempsford R, Allen A, Kelly K, et al. A repeat-dose thorough QY study of inhaled fluticasone furoate/vilanterol combination in healthy subjects. Br J Clin Pharmacol 2014; 77: 466-479.

20 Reddel HK, Barnes DJ. Pharmacological strategies for self-management of asthma exacerbations. Eur Respir J 2006; 28: 182-199.

21 Edwards SJ, Gruffydd-Jones K, Ryan DP. Systematic review and meta-analysis of budesonide/formoterol in a single inhaler. Curr Med Res Opin 2007; 23: 1809-1820.

22 Dinakar C, Oppenheimer J, Portnoy J, et al. Management of acute loss of asthma control in the yellow zone: a practice parameter. Ann Allergy Asthma Immunol 2014; 113: 143-159.

23 Gupta S, Wan FT, Ducharme FM, et al. Asthma action plans are highly variable and do not conform to best visual design practices. Ann Allergy Asthma Immunol 2012; 108: 260-265.e2.

24 Levy ML, Stevenson C, Maslen T. Comparison of short courses of oral prednisolone and fluticasone propionate in the treatment of adults with acute exacerbations of asthma in primary care. Thorax 1996; 51: 1087-1092.

25 Di Franco A, Bacci E, Bartoli ML, et al. Inhaled fluticasone propionate is effective as well as oral prednisone in reducing sputum eosinophilia during exacerbations of asthma which do not require hospitalization. Pulm Pharmacol Ther 2006; 19: 353-360.

26 FitzGerald JM, Shragge D, Haddon J, et al. A randomized, controlled trial of high dose, inhaled budesonide versus oral prednisone in patients discharged from the emergency department following an acute asthma exacerbation. Can Respir J 1999; 7: 61-67.

27 Nana A, Youngchaiyud P, Charoenratanakul S, et al. High-dose inhaled budesonide may substitute for ora therapy after an acute asthma attack. J Asthma 1998; 35: 647-655.

28 Gibson PG, Powell H. Written action plans for asthma: an evidence-based review of the key components. Thorax 2004; 59: 94-99.

29 Foresi A, Morelli MC, Catena E. Low-dose budesonide with the addition of an increased dose during exacerbations is effective in long-term asthma control. On behalf of the Italian Study Group. Chest 2000; 117: 440-446.

30 Oborne J, Mortimer K, Hubbard RB, et al. Quadrupling the dose of inhaled corticosteroid to prevent asthma exacerbations: a randomized, double-blind, placebo-controlled, parallel-group clinical trial. Am J Respir Crit Care Med 2009; 180: 598-602.

31 FitzGerald JM, Boulet L-P, Follows RMA. The CONCEPT trial: a 1-year, multicenter, randomized, double-blind, double-dummy comparison of a stable dosing regimen of salmeterol/fluticasone propionate with an adjustable maintenance dosing regimen of formoterol/budesonide in adults with persistent asthma. Clin Ther 2005; 27: 393-406.

32 Chrystyn H. Do patients show the same level of adherence with all dry powder inhalers? Int J Clin Pract 2005; 59: $19-25$.

33 Rifaat N, Abdel-Hady E, Hasan AA. The golden factor in adherence to inhaled corticosteroid in asthma patients. Egypt J Chest Dis Tuberc 2013; 62: 371-376.

34 Coutts JA, Gibson NA, Paton JY. Measuring compliance with inhaled medication in asthma. Arch Dis Child 1992; 67: $332-333$.

35 Stoloff SW, Stempel DA, Meyer J, et al. Improved refill persistence with fluticasone propionate and salmeterol in a single inhaler compared with other controller therapies. J Allergy Clin Immunol 2004; 113: 245-251.

36 Rau JL. Determinants of patient adherence to an aerosol regimen. Respir Care 2005; 50: 1346-1359.

37 Gupta S, Paolucci E, Kaplan A, et al. Contemporaneous international asthma guidelines present differing recommendations: an analysis. Can Respir J 2016; 3085065.

38 Lugtenberg M, Zegers-van Schaick JM, Westert GP, et al. Why don't physicians adhere to guideline recommendations in practice? An analysis of barriers among Dutch general practitioners. Implement Sci 2009; 4: 5908-5904.

39 Farquhar CM, Kofa EW, Slutsky JR. Clinicians' attitudes to clinical practice guidelines: a systematic review. Med J Aust 2002; 177: 502-506.

40 Lugtenberg M, Burgers JS, Clancy C, et al. Current guidelines have limited applicability to patients with comorbid conditions: a systematic analysis of evidence-based guidelines. PLoS ONE 2011; 6: e25987.

41 Cochrane LJ, Olson CA, Murray S, et al. Gaps between knowing and doing: understanding and assessing the barriers to optimal health care. J Contin Educ Health Prof 2007; 27: 94-102.

42 Gupta S, Rai N, Bhattacharrya $\mathrm{O}$, et al. Optimizing the language and format of guidelines to improve guideline uptake. CMAJ 2016; 188: E362-E368.

43 Wiener-Ogilvie S, Pinnock H, Huby G, et al. Do practices comply with key recommendations of the British Asthma Guideline? If not, why not? Prim Care Respir J 2007; 16: 369-377.

44 van Wersch A, Eccles M. Involvement of consumers in the development of evidence based clinical guidelines: practical experiences from the North of England evidence based guideline development programme. Qual Health Care 2001; 10: 10-16.

45 Ring N, Jepson R, Hoskins G, et al. Understanding what helps or hinders asthma action plan use: a systematic review and synthesis of the qualitative literature. Patient Educ Couns 2011; 85: e131-e143.

46 Cooper V, Metcalf L, Versnel J, et al. Patient-reported side effects, concerns and adherence to corticosteroid treatment for asthma, and comparison with physician estimates of side-effect prevalence: a UK-wide, cross-sectional study. NPJ Prim Care Respir Med 2015; 25: 15026.

47 Moffat M, Cleland J, van der Molen T, et al. Poor communication may impair optimal asthma care: a qualitative study. Fam Pract 2007; 24: 65-70. 